Reprod. Nutr. Develop., 1988, 28 (3 B), 823-824

\title{
Résistance à l'insuline des muscles chez le rat Zucker lors de l'instal- lation de l'obésité
}

\author{
E. DORÉ, L. PÉNICAUD, L. PICON
}

Laboratoire de Physiologie du Développement, UA 307 CNRS, Université Paris 7, 2 Place Jussieu, 75251 Paris Cedex.

Des études récentes, in vivo, ont montré chez les rats obèses Zucker, une résistance à l'insuline dès l'âge de 4 semaines et que ce défaut s'aggrave avec le temps (Pénicaud et al., 1987). Cette insulinorésistance apparaît en premier au niveau musculaire (Pénicaud et al., 1987). Néanmoins, des études réalisées sur le muscle soleus de rats Zucker de 5-6 semaines rapportent des résultats contradictoires en ce qui concerne les perturbations respectives des diverses voies d'utilisation du glucose (Czech et al., 1978; Crettaz et al., 1980). Le but de ce travail était de quantifier les effets de l'insuline sur la glycolyse et la synthèse de glycogène de muscles isolés de rats Zucker obèses ou non.

Les animaux étaient des rats Zucker obèses $(\mathrm{fa} / \mathrm{fa}$ ) ou non ( $\mathrm{Fa} / \mathrm{fa}$ ) âgés de 4 semaines, sevrés depuis une semaine. Le muscle soleus (fibres oxydatives) et l'extensor digitorum longus (EDL, fibres oxydatives et glycolytiques) ont été préparés et incubés selon Crettaz et al. (1980). L'incorporation de ${ }^{3} \mathrm{H}$ dans le glycogène et la production d'eau tritiée à partir de $5{ }^{3} \mathrm{H}$-glucose ont été pris comme index du taux de synthèse de glycogène et de glycolyse (Cuendet et al., 1976). L'insuline était ajoutée au milieu après une préincubation de $20 \mathrm{~min}$. Parmi les concentrations d'insuline étudiées $(100,200$ et $1000 \mu \mathrm{U} / \mathrm{ml})$ les deux plus faibles avoisinent l'insulinémie basale des témoins et des obèses, la plus forte permet d'obtenir l'effet maximal de l'hormone.

Les animaux obèses ont une insulinémie $(159 \pm 8 \mu \mathrm{U} / \mathrm{ml})$ et une glycémie $(104 \pm 1 \mathrm{mg} / \mathrm{dl})$ significativement supérieures à celles des témoins $(82 \pm 9 \mu \mathrm{U} / \mathrm{ml}, \mathrm{p}<0,01$ et $93 \pm 2 \mathrm{mg} / \mathrm{dl}, \mathrm{p}<0,01)$. Le poids des soleus est identique dans les deux groupes, l'EDL étant plus lourd chez les témoins $(26,6 \pm 0,7 \mathrm{mg})$ que chez les obèses $(23,7 \pm 0,8 \mathrm{mg}, \mathrm{p}<0,01)$. Les résultats montrent une légère diminution de la sensibilité à l'insuline sans changement de la réponse maximale pour le métabolisme total (glycolyse + glycogénogenèse) chez les animaux obèses (tabl.). Cette diminution est due en majeure partie à une diminution de la sensibilité à l'insuline de la glycolyse. La concentration en insuline nécessaire pour produire la moitié de la réponse maximale est de $160 \mu \mathrm{U} / \mathrm{ml}$ pour le soleus et de $260 \mu \mathrm{U} / \mathrm{ml}$ pour l'EDL chez les non-obèses et de 310 et $460 \mu \mathrm{U} / \mathrm{ml}$ respectivement chez les obèses, les réponses maximales étant identiques dans les deux groupes et pour les deux muscles. Ces données rejoignent celles établies précédemment pour le soleus par Crettaz et al. (1980).

Pour l'incorporation du glucose dans le glycogène, la sensibilité à l'insuline des muscles des obèses et de ceux des témoins est identique. Les différences en valeur absolue rapportées dans le tableau 1 sont dues à la différence observée en 
TABLEAU 1

Effet de l'insuline sur la glycolyse, I'incorporation du g/ucose dans le glycogène et le métabolisme total du glucose dans le soléus et I'EDL de rats Zucker obèses ou non âgés de 4 semaines. Le nombre de cas est indiqué entre parenthèses. ${ }^{*} p<0,05,{ }^{* *} p<0,01,{ }^{* * *} p<0,001$.

\begin{tabular}{|c|c|c|c|c|c|}
\hline Muscles & Animaux & $\begin{array}{l}\text { Insuline } \\
(\mu \mathrm{U} / \mathrm{ml})\end{array}$ & $\begin{array}{l}\text { Glycolyse } \\
(\mathrm{nmol} / \mathrm{mg} / \mathrm{h}\end{array}$ & $\begin{array}{l}\text { Glycogénogenèse } \\
\text { (nmol/mg/h }\end{array}$ & $\begin{array}{l}\text { Métabolisme } \\
(\mathrm{nmol} / \mathrm{mg} / \mathrm{h})\end{array}$ \\
\hline \multirow{8}{*}{ Soleus } & \multirow{4}{*}{$\mathrm{Fa} / \mathrm{fa}$} & 0 & $5.8+0.5$ & $0,83+0,13$ & $6.6+0.4$ \\
\hline & & 100 & $8,0 \pm 0,4$ & $1,38 \pm 0,27$ & $9,4 \pm 0,2$ \\
\hline & & 200 & $12,7 \pm 0,6$ & $1,64 \pm 0,24$ & $14,4 \pm 0,6$ \\
\hline & & 1000 & $15,5 \pm 1,6$ & $2,16 \pm 0,39$ & $17,7 \pm 1,5$ \\
\hline & \multirow{4}{*}{$\mathrm{fa} / \mathrm{fa}$} & 0 & $5.7+0.6$ & $0.45+0.09^{*}$ & $61+0.6 \quad(16)$ \\
\hline & & 100 & $73+1,1$ & $0,84+0,2$ & $8,2 \pm 1,1$ \\
\hline & & 200 & $8,9 \pm 0,4^{* * *}$ & $1,48 \pm 0,24$ & $10,4 \pm 0,6^{* *}(12)$ \\
\hline & & 1000 & $14,0 \pm 1,1$ & $1,21 \pm 0,16^{*}$ & $15,2 \pm 1,2$ \\
\hline \multirow{8}{*}{ EDL } & \multirow{8}{*}{$\mathrm{fa} / \mathrm{fa}$} & 0 & $5,8 \pm 0,6$ & $0,78 \pm 0,09$ & $6,6 \pm 0,6$ \\
\hline & & 100 & $6,1 \pm 0,7$ & $1,03 \pm 0,26$ & $8,1 \pm 0,2$ \\
\hline & & 1000 & $8,3 \pm 0,6$ & $1,41 \pm 0,33$ & $9,7 \pm 0,5$ \\
\hline & & & & & \\
\hline & & 0 & $5,5 \pm 0,5$ & $0,34 \pm 0,007^{* * *}$ & $5,8 \pm 0,5 \quad(16)$ \\
\hline & & 100 & $6,0 \pm 0,3$ & $0,26 \pm 0,03^{* *}$ & $6,3 \pm 0,4$ \\
\hline & & 200 & $5,8 \pm 0,5^{*}$ & $0,76 \pm 0,16$ & $6,6 \pm 0,5^{* *}(12)$ \\
\hline & & 1000 & $8,8 \pm 1,3$ & $1,19 \pm 0,32$ & $9,9 \pm 1,2$ \\
\hline
\end{tabular}

absence d'insuline, fait déjà décrit par certains auteurs (Cuendet et al., 1976). Lorsque les résultats sont exprimés en \% de l'effet maximal, l'effet de l'insuline est identique dans les deux groupes.

En conclusion, ces données montrent une baisse de la sensibilité à l'insuline dans les muscles de rats obèses, cette baisse affectant principalement la voie de la glycolyse. Néanmoins, même au niveau de la synthèse de glycogène, l'utilisation du glucose est moins importante chez les obèses. De plus, ces données confirment, in vitro, une différence de sensibilité à l'insuline suivant le type de muscle considéré, le muscle oxydatif (soleus) étant plus sensible que le muscle glycolytique (EDL) (James et al., 1985).

Nous remercions A. Leturque et $\mathrm{P}$. Maulard pour leurs conseils sur la mise au point des techniques.

Crettaz M., Prentki M., Zaninetti D., Jeanrenaud B., 1980. Biochem. J., 186, 525-534.

Cuendet G., Loten E., Jeanrenaud B., Renold A., 1976. J. clin. Invest., 58, 1078-1088.

Czech M., Richardson D., Becker S., Walters C., Gitomer W., Heinrich J., 1978. Metabolism, 27, 1967-1981.

James D., Jenkins A., Kraegen E., 1985. Amer. J. Physiol., 248, 567-574.

Pénicaud L., Ferré P., Terretaz J., Kinebanyan M. F., Leturque A., Doré E., Girard J., Jeanrenaud B., Picon L., 1987. Diabetes, 36, 626-631. 